\title{
INOVAÇÃO EM SERVIÇOS DE SOFTWARE: QUAL O PAPEL DOS CLIENTES, DA PESQUISA E DO DESENVOLVIMENTO?
}

\section{INNOVATION IN SOFTWARE SERVICES: WHICH ROLE FOR CLIENTS, RESEARCH AND DEVELOPMENT?}

Timóteo Guimarães da Conceição Programa de Pós-Graduação em Administração - PPGA / Universidade de Brasília - UnB, BACEN - Banco Central do Brasil timoguimaraes@gmail.com

Marina Figueiredo Moreira

Programa de Pós-Graduação em Administração - PPGA / Universidade de Brasília - UnB marinamoreira.adm@gmail.com

Submissão: $12 / 03 / 2015$

Aprovação: 24/11/2016 


\title{
RESUMO
}

Este estudo investigou o papel desempenhado por atividades de P\&D e clientes nas práticas de gestão da inovação em empresas desenvolvedoras de serviços de software no Distrito Federal (DF). Realizou-se estudo de casos múltiplos com dez unidades de análise com coleta de evidências por entrevistas semiestruturadas e análise de conteúdo por codificação temática. Os resultados permitiram caracterizar o processo empírico de gestão da inovação efetivamente adotado nos casos como sendo de baixa formalização, baixa estruturação e com altos índices de retrabalho, sendo muitas vezes disparado por fontes não tradicionais. Ressalta-se como principal contribuição do artigo o apontamento da dissonância entre o discurso dos entrevistados sobre a importância de clientes e P\&D e o efetivo papel desempenhado por esses inputs nas práticas organizacionais. Conclui-se, assim, que os modelos teóricos embasam o discurso dos entrevistados; mas não suas práticas organizacionais.

Palavras-chave: Inovação; Inovação em serviços; Serviços de software; Pesquisa e Desenvolvimento; Modelos de Inovação.

\begin{abstract}
The present study investigated the role played by $R \& D$ activities and by customers in innovation management practices in companies that develop software services in the Brazilian Federal District. We conducted a multiple case study with ten units of analysis with evidence collection by semi-structured interviews and content analysis by thematic coding. Results allowed the characterization of the empirical process of innovation management effectively adopted in the cases as being associated with low formalization, low structure and high levels of reworking. Innovation process is often triggered by non-traditional sources. We highlight as the paper's main contribution the pointing of the dissonance between the discourse of the respondents regarding the importance of customers and $R \& D$ and the effective role played by these inputs in organizational practices. We conclude, therefore, that the theoretical models base the discourse of the respondents; but not their organizational practices.
\end{abstract}

Keywords: Innovation; Service Innovation; Software Services; Research \& Development; Clients. 


\section{Introdução}

No contexto de uma economia crescentemente baseada em conhecimento, dois fenômenos têm ocupado lugar de destaque entre as novas metas empresariais: a aprendizagem e a inovação (FORAY; LUNDVALL, 1998; BESSANT; TIDD, 2009; NELSON; WINTER, 2005). Desde a década de 1950, a visão inicial da diferenciação mercadológica como caminho para obtenção de vantagem competitiva por uma empresa (PORTER, 2008) veio gradualmente dando espaço a abordagens que priorizam a capacidade de inovação como real preditor do sucesso empresarial.

Com a consolidação dos papeis da inovação, tecnologia e conhecimento na dinâmica produtiva pós-fordista, uma questão emerge: como as empresas podem aumentar sua capacidade de inovação? Respostas a esta demanda se apresentam sob o prisma de ações de gestão do processo da inovação, o que exige adotar modelos de gestão e produção eficientes e voltados à geração de soluções de potencial inovador. A inovação, por se configurar em um processo não-isolado, exige gerenciamento integrado junto a outras atividades da empresa (TIDD; BESSANT; PAVITT, 2008), entre as quais se destacam aquelas voltadas ao desenvolvimento de novos bens ou serviços e à sua promoção junto ao mercado consumidor notadamente reconhecidas como as áreas de Desenvolvimento e de Marketing.

O papel dessas áreas é bem conhecido no contexto dos modelos de gestão da inovação desenvolvidos por consecutivas gerações de pesquisadores ao longo das últimas décadas. Sabese que Pesquisa e Desenvolvimento (P\&D) e clientes são os inputs mais fortemente reconhecidos pela literatura como associados à geração de inovações (VIOTTI; MACEDO, 2003; DORNER; GASSMANN; GEBAUER, 2011; OKE, 2007; GUIMARÃES, 2011; VASCONCELLOS; MARX, 2011; HANSEN; BIRKINSHAW; 2007; BREM; VOIGT, 2009). Sabe-se, também, que a P\&D se organiza em muitas empresas como atividade de geração ou aumento de estoque de conhecimentos, fases que antecedem o próprio processo de inovação. A interação com os clientes como input dos processos de inovação, por sua vez, também é reconhecida pela teoria. Mas até que ponto há correspondência entre o que se sabe sobre o processo de inovação e as práticas diárias das firmas? As empresas realmente reconhecem a importância da $\mathrm{P} \& \mathrm{D}$ e dos clientes em suas práticas de gestão da inovação? O papel atribuído a P\&D e aos clientes nos modelos teóricos como fontes indutoras de inovações corresponde, realmente, ao papel desempenhado por esses dois elementos no dia-a-dia das empresas?

Especialmente no tocante à gestão da inovação, um setor tem recebido destaque por sua participação econômica. Trata-se do setor de serviços, que tem ocupado posições majoritárias na composição do PIB das economias mais desenvolvidas desde a década de 1950 (GADREY, 2001). No Brasil, o setor de serviços tem chamado a atenção de pesquisadores, empresários e formuladores de políticas públicas (VARGAS et al., 2010), tendo se configurado como o maior receptor dos ingressos brutos de Investimento Externo Direto (IED) até setembro de 2007, com $47,3 \%$ do total, frente a 39,3\% destinados ao setor industrial (KUBOTA, 2009). Com a consolidação do setor de serviços como estrutural na economia brasileira e, ainda, com o imperativo de inovar imposto às empresas nacionais, alguns questionamentos pertinentes emergem: como tem se dado o processo de gestão da inovação nas empresas de serviços no Brasil? As empresas de serviços reconhecem o papel da P\&D e dos clientes em sua gestão interna de inovação?

Para contribuir com a elucidação destes questionamentos, este estudo investiga o papel desempenhado pelas atividades de $\mathrm{P} \& \mathrm{D}$ e pelos clientes na geração de inovações em empresas do setor de serviços, com foco específico para o segmento dos serviços de software, tradicionalmente reconhecidos como altamente dependentes de inovações. Adota-se, assim, a 
seguinte pergunta de pesquisa: que papel desempenham P\&D e clientes no processo de gestão da inovação de empresas de serviços de software atuantes no Distrito Federal?

Este trabalho desenvolve três seções teóricas iniciais que discutem o processo de gestão da inovação em serviços com especial foco no exame dos modelos para a gestão da inovação tradicionalmente relatados na teoria. Em particular, explora-se o papel desempenhado por dois vetores de inovações - atividades de P\&D e clientes. Na sequência, descrevem-se os métodos utilizados para a realização da pesquisa de campo, que consiste em um estudo de casos múltiplos com dez unidades de análise. Por fim, apresentam-se as descrições e análises dos relatos obtidos a partir das entrevistas com especialistas dos casos, a partir das quais se elaboram as conclusões.

\section{Modelos para o processo de gestão da inovação: o que 70 anos de teoria nos revelam?}

O fenômeno da inovação tem sido objeto de estudos nos campos da Economia e, mais recentemente, da Administração, desde o início do século XX, com a proposição inicial de Schumpeter para a definição do conceito. Inicialmente, Schumpeter (1997, p. 95) define a inovação a recombinação de recursos pré-existentes de forma a gerar valor até então inédito. Ao diferenciar invenção de inovação com o propósito de estabelecer os limites desta última, o autor afirma que sua ocorrência exige que uma invenção seja levada à prática e, adicionalmente, adquira relevância econômica após sua introdução no mercado. Esclarece-se, assim, que a ação de lançar invenções no mercado é o caminho para o teste de novas tecnologias e, caso se confirme a premissa inicial de geração de valor pelo mercado, tem-se o surgimento de uma inovação.

Ao longo das últimas décadas do século XX, o aumento do nível de conhecimento sobre o fenômeno da inovação levou à proposição de consecutivos modelos representativos de seu processo, permitindo-nos traçar uma representação histórica dessa evolução. Essa trajetória se inicia com a $1^{a}$ Geração de modelos para a gestão da inovação, surgindo por volta da década de 1950 e se mantendo até meados de 1960 (ROTHWELL, 1994). Neste período, as economias de mercado registraram elevadas taxas de crescimento em função da expansão industrial e o processo de inovação na indústria era visto como uma progressão linear (Figura 1), com foco no papel desempenhado pelas atividades de $\mathrm{P} \& \mathrm{D}$ nas empresas. $\mathrm{P} \& \mathrm{D}$ eram admitidas como os próprios indicadores do processo de inovação, entendendo-se que a inovação só surgiria como resultado dessas atividades. $\mathrm{P} \& \mathrm{D}$ seriam, portanto, os iniciadores do processo de desenvolvimento de uma inovação, ao qual se seguiriam as etapas de pesquisa aplicada, produção e difusão (GODIN, 2006). Rothwell (1994) apresenta uma versão do chamado Modelo Linear de Inovação, que representa graficamente a premissa estabelecida no contexto da abordagem Technology Push, que admite as atividades de Pesquisa como representantes dos investimentos científicos para a geração de novos produtos (KLINE; ROSENBERG, 1986). Este modelo - apresentado na Figura 1 - e a abordagem que ele representa demarcam, portanto, o papel absoluto designado às atividades de $\mathrm{P} \& \mathrm{D}$ como responsáveis unilaterais pelo surgimento de inovações.

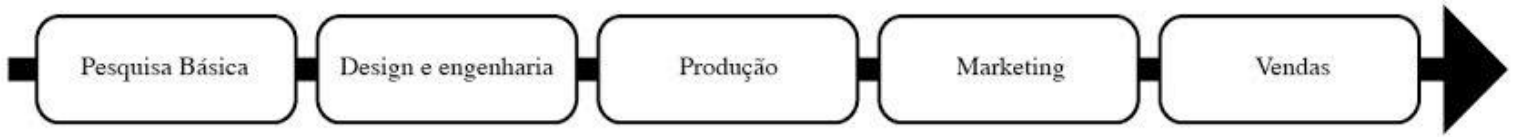

Figura 1 - Technology Push (Primeira Geração)

Fonte: adaptado de Rothwell (1994, p. 8). 
Objeto de muitas críticas apontando seu caráter reducionista em função do posicionamento exclusivo das atividades de $\mathrm{P} \& \mathrm{D}$ como preditoras de inovações, o Modelo Linear e a abordagem Technology Push entram em declínio, dando origem à chamada $2^{\mathrm{a}}$ Geração de modelos para o processo de inovação. Na segunda metade da década de 1960, a ênfase dos investimentos deixou de se focar em novos produtos e políticas tecnológicas expansionistas e se voltou a mudanças tecnológicas com maior racionalidade, gerando crescimento nos investimentos em marketing e maior disputa por mercado (ROTHWELL, 1994). As demandas dos consumidores se tornaram fontes de ideias para orientar as atividades de P\&D que, por sua vez, passaram a desempenhar papel reativo às demandas. Esse novo mapeamento do processo de gestão da inovação corresponde à premissa da Market-pull, abordagem apontada por Rothwell (1994) como responsável pela segunda geração de modelos. Tem-se um exemplo deste modelo na Figura 2.

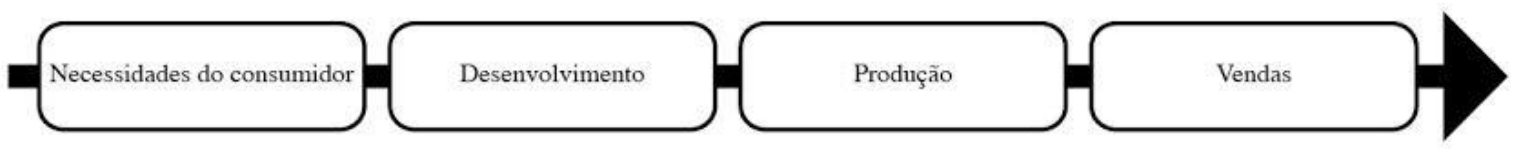

Figura 2 - Market Pull (Segunda Geração)

Fonte: adaptado de Rothwell (1994, p. 9).

Os dois primeiros modelos compartilham uma visão comum em relação à inovação: ambos a entendem como uma "sequência linear de atividades" (TIDD; BESSANT; PAVITT, 2008, p. 95), razão pela qual são alvos de críticas (NIOSI, 1999), dando origem, em 1970, à $3^{\text {a }}$ Geração de modelos. Esse período é marcado por duas crises de petróleo (em 1973 e 1979), elevadas taxas de inflação e baixo crescimento, além de desemprego estrutural, obrigando as empresas a buscar inovações bem sucedidas para reduzir a incidência de falhas e o desperdício.

Neste período, uma série de estudos detalhados sobre a inovação é publicada e, pela primeira vez, um processo de inovação bem sucedido foi modelado. Estes estudos mostraram que os dois modelos anteriores representavam extremos e, mais que isso, eram falíveis para representar um processo abrangente, que envolvia interação entre capacidade tecnológica e demanda de mercado. Surgiu, então, um modelo interativo - ou coupling - que apresentava uma série de estágios interativos e interdependentes. Entendia-se que o sucesso ou fracasso de uma inovação raramente poderiam ser explicados por um ou dois fatores, mas por uma série de procedimentos interconectados, conforme Figura 3.

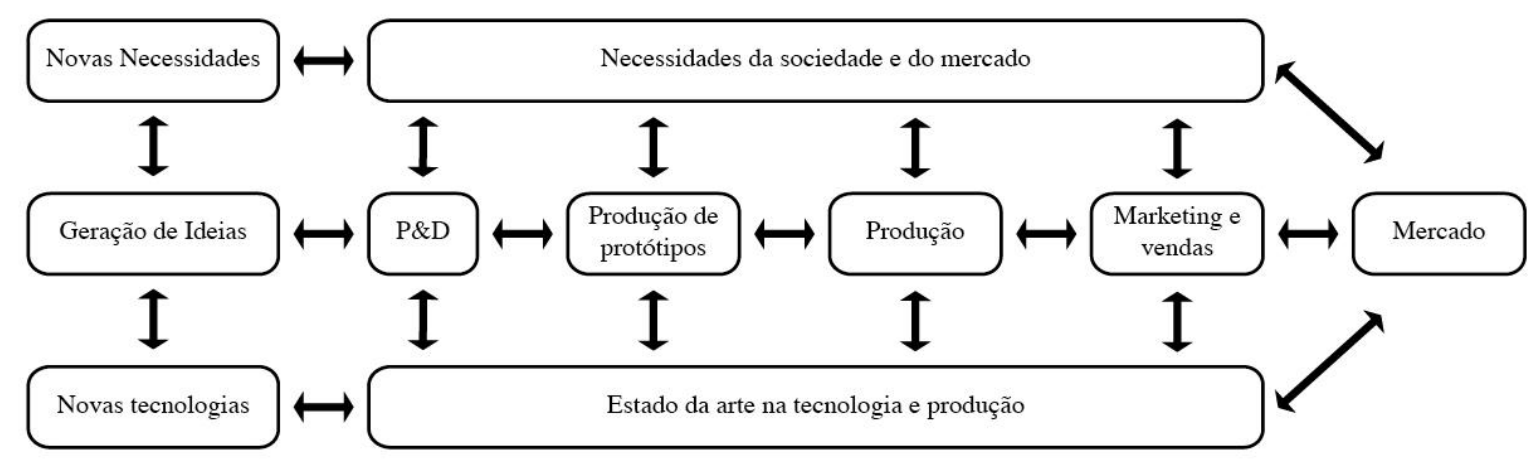

Figura 3 - Modelo de Inovação Coupling (Terceira Geração) 
Fonte: adaptado de Rothwell (1994, p. 10).

O início da década de 1980 registra o surgimento de uma nova geração de modelos. Tratase da $4^{\mathrm{a}}$ Geração, que surge em um momento de recuperação econômica das empresas e é marcada por valorização da acumulação tecnológica, foco na estratégia de manufatura e aumento das alianças estratégicas entre empresas. As empresas japonesas, base do modelo de inovação da quarta geração, utilizavam integração - que envolvia seus fornecedores no processo de desenvolvimento de novos produtos - e o desenvolvimento paralelo - que envolvia diferentes departamentos internos no trabalho simultâneo (paralelo) ao invés de sequencial (em série), conforme Figura 4. Ao final da década de 1980, tem-se o surgimento da $5^{\text {a e última }}$ geração modelos, que veem a inovação como um processo multifatorial que exige grande integração em níveis intra e inter corporativos, sendo crescentemente fomentado por trabalho em rede baseado em tecnologias da informação.

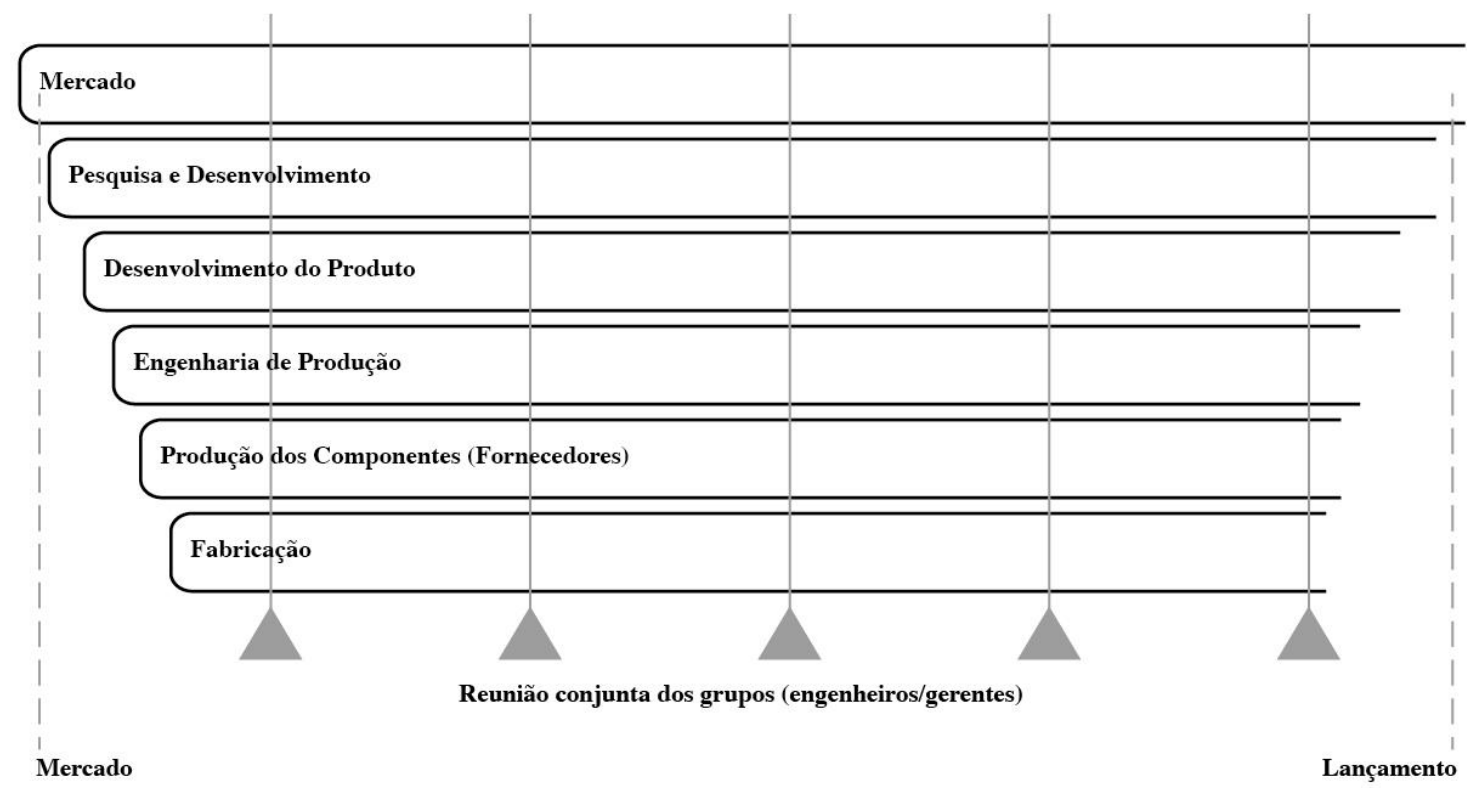

Figura 4 - Exemplo do Processo Integrado de Inovação (Quarta Geração)

Fonte: adaptado de Rothwell (1994, p. 12).

O que podemos inferir a partir dessa trajetória de evolução dos modelos representativos do processo de gestão da inovação nas empresas? Em primeiro lugar, vê-se que a própria visão associada ao processo de inovação se alterou. Inicialmente tido como um processo linear nos modelos de $1^{\mathrm{a}}$ e $2^{\mathrm{a}}$ geração, essa visão rapidamente se alterou de forma permanente para os modelos de $4^{\mathrm{a}}$ e $5^{\mathrm{a}}$ gerações. O caráter não-linear do processo e as relações de interdependência entre etapas, atores e áreas vão se tornando mais evidentes com a própria evolução dos modelos, inicialmente sob perspectiva intrafirma e, posteriormente, com foco também em elementos externos às firmas. Em segundo, é possível analisar quais atores, elementos e etapas são admitidos para o processo de inovação ao longo das várias evoluções relatadas. Os primeiros modelos, que compreendem a $1^{\mathrm{a}}$ e a $2^{\mathrm{a}}$ geração de Rothwell (1994), apontam, respectivamente, as atividades de P\&D e os clientes como "disparadores" do processo de inovação. Ou seja: caberia a estes dois elementos a função de provocar o processo de desenvolvimento de inovação nas firmas. A $3^{\text {a }}$ geração, por sua vez, revela maior complexidade nas inter-relações e nas etapas 
envolvidas no processo de inovação, mas não extingue a participação dos dois elementos cruciais - P\&D e clientes. Essa tendência se mantém para os modelos de $4^{\mathrm{a}}$ e $5^{\mathrm{a}}$ gerações, que incorporam perspectivas sistêmicas e setoriais aumentando a complexidade dos modelos para o processo de inovação sem, no entanto, diminuir o papel exercido por P\&D e clientes. Essa análise continuada dos modelos ao longo de cinco gerações revela, portanto, que os dois elementos mapeados desde os primeiros esforços teóricos se mantiveram reconhecidos como indutores do processo de inovação, justificando, portanto, a proposta de pesquisa delineada para este estudo.

\section{Gestão da inovação em serviços}

Os modelos para a gestão da inovação apresentados por Rothwell (1994) se propõem a relatar a realidade empírica observada nas firmas. Inicialmente voltados para empresas dos setores industriais, esses modelos revelam sua correspondência histórica às décadas de $1950 \mathrm{e}$ 1960, em que a maior parte da produção econômica nos países desenvolvidos era gerada em empresas do setor industrial. A visão inicial relatada no Modelo Linear, que posiciona as atividades de $\mathrm{P} \& \mathrm{D}$ como responsáveis exclusivas pela geração de inovações, representa a própria dinâmica de geração de novos produtos na lógica produtiva industrial. Nessa perspectiva, caberia às atividades de Pesquisa - representantes diretas da Ciência - a tarefa de buscar novos materiais, fontes de matérias-primas ou tecnologias para que as firmas, então, as transformassem em produtos novos a serem lançados no mercado. Mas o que ocorreria no caso de firmas que não se voltam à produção de bens tangíveis, mas à prestação de serviços?

Inicialmente admitidas como incapazes de gerar inovações genuínas e tidas apenas como disseminadoras de inovações originárias da indústria (BARRAS, 1986), as empresas de serviços passaram por trajetória notória no tocante à sua participação econômica nas economias desenvolvidas. De forma concomitante ao declínio do modelo de produção fordista após a década de 1940 - e, naturalmente, junto ao próprio declínio do setor industrial -, as empresas de serviços foram gradativamente aumentando sua participação econômica a partir da década de 1950. Esse processo lhe garantiu, então, a posição majoritária hoje ocupada pelo setor de serviços no Produto Interno Bruto das principais economias mundiais, em que o setor de serviços responde por taxas acima de 75\% da produção total (GADREY, 2001; RUBALCABA, 2007).

Em empresas de serviços, sabe-se que a gestão da inovação guarda peculiaridades. Evidencia-se o papel central desempenhado pelo cliente no processo de inovação - razão pela qual modelos compreensivos quanto a esse papel costumam ter maior uso em estudos que investigam serviços (GALLOUJ, 2002). O papel da P\&D, por sua vez, também não é ignorado em atividades de serviços. Sabe-se que neste setor os processos de P\&D se mostram diferenciados em relação ao observado nas empresas industriais, mas certamente presentes, embora menos formalizados (SAWATANI; FUJIGAKI, 2015). E quanto à ocorrência de inovações? Sabe-se que este processo possui peculiaridades quando comparado ao processo industrial. Registram-se, até hoje, três abordagens para o estudo e a compreensão da inovação em serviços: a) uma voltada à replicação dos modelos e métricas industriais para as análises em serviços; b) uma para estabelecer critérios específicos e distintos para as inovações em serviços; c) uma chamada integradora, que unifica os esforços teóricos sem dissociar inovações em bens ou serviços (GALLOUJ; SAVONA, 2008).

$\mathrm{Na}$ abordagem integradora, destaca-se o modelo inicialmente proposto por Gallouj e Weinstein (1997) e posteriormente aperfeiçoado em Gallouj (2002). Trata-se de um modelo para a representação de produtos - bens ou serviços, com aplicação indistinta - sob o formato de vetores de características técnicas materiais ou imateriais e competências de prestadores e 
clientes. O modelo é apresentado na Figura 5 e defende que a inovação seria o resultado de alterações na dinâmica vetorial capazes de afetar um ou mais elementos de um ou mais vetores de características (técnicas ou materiais) ou, ainda, de competências. Essas inovações podem ser planejadas ou intencionais - resultando de investimentos em P\&D - ou não intencionais resultando de processos de aprendizagem dos participantes (clientes ou prestadores).

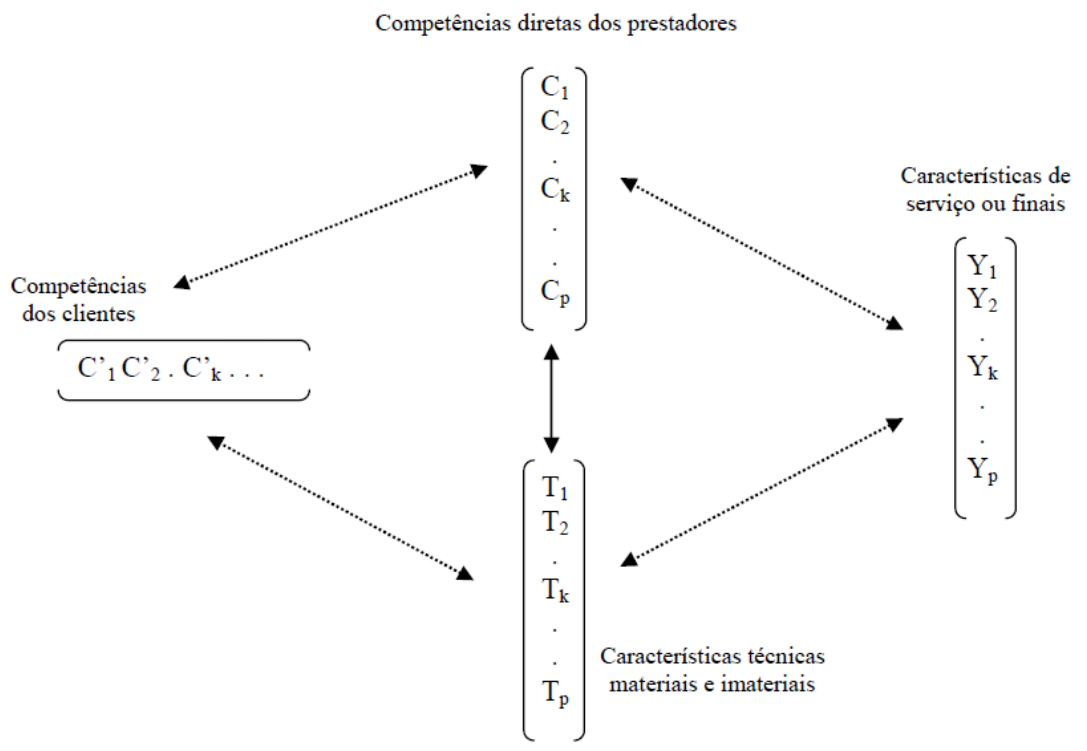

Figura 5 - Prestação do Serviço como um Conjunto de Vetores Fonte: adaptado de Gallouj (2002, p. 58).

Campos, Nicolau e Simioni (2002) mostram que a prestação do serviço especializado, a exemplo dos serviços de software, exige interação entre fornecedores e clientes, além de esforços de P\&D para oferecer soluções de qualidade na interface entre conhecimento de informática e do setor específico, além de proximidade com o cliente-usuário e ampla assistência técnica antes da instalação do programa (treinamentos) e após a instalação (suporte). Trata-se de uma peculiaridade que reforça, justamente, uma das características definidoras dos serviços: o aspecto relacional, ou seja, a dependência de interação cliente-consumidor como caminho para a ocorrência do serviço (SALERNO, 2001) - também entendida como coprodução (GADREY; GALLOUJ; WEINSTEIN, 1994). Entende-se, assim, que a produção de um serviço envolve desenvolver uma solução para um problema (um tratamento, uma operação) colocando à disposição do cliente as capacidades e competências (humana, tecnológica, organizacional) da firma para gerar a solução mais adequada e precisa. Essas soluções mais tanto mais individualizadas quanto maior for o nível de interação entre prestador e cliente.

\section{P\&D e clientes como inputs de inovação em serviços}

Neste estudo, interessa-nos especialmente investigar o papel exercido por atividades de $\mathrm{P} \& \mathrm{D}$ e por clientes. Especialmente em relação à $\mathrm{P} \& \mathrm{D}$, sabe-se que se organiza como atividade de geração e aumento do estoque de conhecimentos, fases que antecedem o processo de inovação. Em serviços, a P\&D ocorre normalmente em departamentos chamados de "desenvolvimento empresarial", "tecnologia empresarial" ou, ainda, na função de marketing. Em empresas de pequeno porte, por sua vez, essas atividades normalmente ocorrem no design ou no "suporte técnico" (TIDD; BESSANT; PAVITT, 2008, p. 225). Assim, tradicionalmente, 
a P\&D em serviços representa um dos meios mais utilizados por organizações elevar seus estoques de conhecimento (NIOSI, 1999).

Mas o que se sabe, em termos teóricos, sobre o papel de P\&D no processo de inovação? Os primeiros modelos de inovação, descritos na $1^{\mathrm{a}}$ Geração (technology-push) de Rothwell (1994), eram basicamente lineares, com origem no conhecimento gerado por pesquisa básica (laboratório) que, uma vez desenvolvida, chegava à firma, que o transformava em produtos destinados ao mercado (GUIMARÃES, 2011). Esse processo é descrito por Viotti e Macedo (2003) e apresentado de forma estruturada, conforme Figura 6.

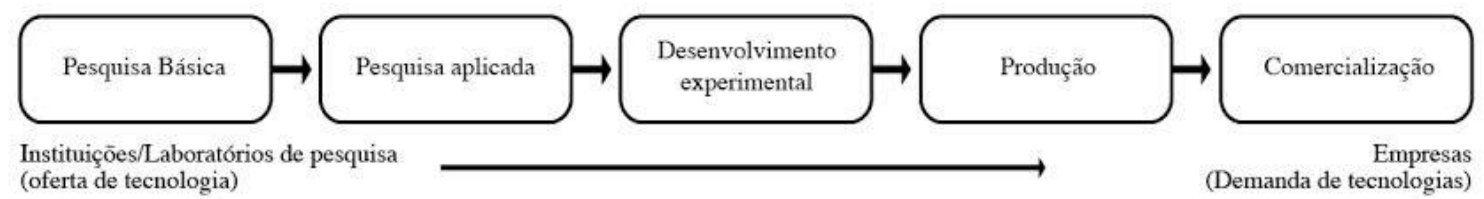

Figura 6 - Modelo Linear de Inovação

Fonte: adaptado de Viotti e Macedo (2003, p. 55).

O modelo linear pressupõe a existência de relação mais ou menos direta entre as quantidades e qualidades dos insumos utilizados em P\&D e os resultados em termos de inovação tecnológica e desempenho econômico. Esse processo ocorreria por intermédio de etapas mais ou menos fixas e sequenciais que resultariam em: pesquisa básica, em que seria gerado o conhecimento científico sobre o qual poderia ser desenvolvida a pesquisa aplicada e, posteriormente, desenvolvimento experimental (VIOTTI; MACEDO, 2003). Como afirma Guimarães (2011), sustentava-se que conhecimento e inovação seriam processos isolados. Por fim, a invenção resultante dos esforços de $\mathrm{P} \& \mathrm{D}$ seria incorporada à produção, comercializada e, assim, transformada em inovação (VIOTTI; MACEDO, 2003). Brem e Voigt (2009) ressaltam que, neste modelo, a existência prévia de demanda para o novo produto é irrelevante.

Em relação ao próprio conceito de $\mathrm{P} \& \mathrm{D}$, é possível partir da definição do Manual Frascati (OCDE, 2002, p. 30), que as define como aquelas "criativas realizadas sob uma base sistemática, com o objetivo de aumentar o estoque de conhecimento, incluindo o conhecimento do homem, da cultura e da sociedade, e a utilização deste estoque de conhecimento na criação de novas aplicações". Considera-se que P\&D é constituída por pesquisa básica, aplicada e desenvolvimento experimental. A primeira se refere ao trabalho experimental ou teórico desenvolvido para adquirir conhecimento por meio de fatos observáveis, sem visar qualquer aplicação ou uso. A pesquisa aplicada diferencia-se da primeira por possuir propósito ou objetivo prático. Já o desenvolvimento experimental constitui-se em trabalho sistemático, que visa, a partir do conhecimento obtido com as pesquisas, promover importantes melhorias em materiais, produtos, processos ou serviços (OCDE, 2002). Como os projetos podem ser desenvolvidos por outros departamentos que não os específicos de $\mathrm{P} \& \mathrm{D}$, confirma-se que a realização dessas atividades não segue limites organizacionais, podendo ocorrer em departamentos inicialmente não previstos - especialmente no caso dos serviços.

Para explorar o papel da $\mathrm{P} \& \mathrm{D}$ em serviços, cabe recuperar a explicação atribuída à dinâmica da inovação em serviços apresentada na Figura 5, que demonstra que a inovação em serviços pode ser definida de acordo com mudanças que afetem um ou mais elementos de um ou mais vetores de características ou competências (GALLOUJ, 2002) - naturalmente, desde que sejam capazes de gerar valor, por se tratar se teoria neo-schumpeteriana (NELSON; WINTER, 2004). A inovação poderia se apresentar, ainda, como a evolução ou variação, saída ou entrada de um ou mais elementos; e a associação, dissociação ou formatação de um ou mais elementos. Isto pode ocorrer de forma intencional ou planejada, como resultado de $\mathrm{P} \& \mathrm{D}$, por 
exemplo, ou não intencionais, a partir de um aprendizado inercial por parte dos agentes envolvidos (GALLOUJ; SAVONA, 2008).

No setor de serviços, a maior parte dos investimentos se refere à produção de conhecimentos vinculados a novos conceitos organizacionais e de marketing, novas interfaces com o cliente, novas formas de entrega e combinações de serviços e produtos. Esses investimentos incluem esforços relacionados à $\mathrm{P} \& \mathrm{D}$ que nem sempre são facilmente identificados e mensurados, tendo em vista a intangibilidade de seus produtos (RENESER, 2006). Especialmente em relação ao papel da $P \& D$ em serviços de software, cabe considerar alguns dados. Segundo levantamento da Softex (2009, p. 97), das 2.197 empresas participantes da PINTEC 2005 que implementaram inovações no período 2003-2005, 1.829 realizaram dispêndios com as atividades inovativas em 2005. A atividade inovativa mais realizada foi a "aquisição de máquinas e equipamentos" (76\%), além de "atividades internas de P\&D", "treinamento" e "aquisição de software". Este estudo atesta, ainda, que os esforços em P\&D para inovar ocorrem no âmbito da própria empresa, sendo reduzido o número de empresas que "adquiriram conhecimentos externos" (24\%) ou "realizaram P\&D externa" (4\%). Por fim, cabe ressaltar que as empresas gastaram, em 2005, R \$ 1,6 bilhão em atividades voltadas à inovação. Esse valor representa 5,2\% da receita líquida total da IBSS (Indústria Brasileira de Software e Serviços de TI) em 2005. Entre as atividades inovativas, as "atividades internas de P\&D" foram as que mais consumiram recursos financeiros (39,2\% do total de dispêndios), segundo a Softex (2009).

A Figura 7 lista as fontes de informação internas e externas que vêm sendo utilizadas pelas empresas da indústria brasileira de software e serviços de TI e pelos demais setores cobertos pela PINTEC 2005. As proporções mencionadas referem-se ao percentual de empresas inovadoras que avaliou como de alta relevância as fontes de informação utilizadas. Na IBSS, as "redes de informação informatizadas" e os "clientes e consumidores" são fontes de informação utilizadas e avaliadas de importância alta pelas empresas do setor de software e serviços de TI. Para as empresas de desenvolvimento de software, o "departamento de P\&D" também foi considerado fonte importante de informação (SOFTEX, 2009, p. 106). 


\begin{tabular}{|c|c|c|c|c|c|c|}
\hline & \multirow{2}{*}{ Fontes de Informação } & \multicolumn{2}{|c|}{ Indústria } & \multicolumn{3}{|c|}{ Serviços } \\
\hline & & Extrativa & Transformação & Teleconunicações & IBSS & P\&D \\
\hline \multirow{2}{*}{$\begin{array}{l}\text { Fontes } \\
\text { internas }\end{array}$} & Departamento de P\&D & $1,4 \%$ & $7,5 \%$ & $23,9 \%$ & $27,4 \%$ & $85,4 \%$ \\
\hline & Outras áreas & $38,2 \%$ & $41,7 \%$ & $59,4 \%$ & $45,2 \%$ & $26,8 \%$ \\
\hline \multirow{12}{*}{$\begin{array}{l}\text { Fontes } \\
\text { externas }\end{array}$} & Outra empresa do grupo & $1,6 \%$ & $3,1 \%$ & $18,9 \%$ & $5,4 \%$ & $12,2 \%$ \\
\hline & Fornecedores & $43,6 \%$ & $40,2 \%$ & $55,0 \%$ & $36,2 \%$ & $19,5 \%$ \\
\hline & Clientes ou consumidores & $35,4 \%$ & $42,8 \%$ & $32,2 \%$ & $47,7 \%$ & $56,1 \%$ \\
\hline & Concorrentes & $31,9 \%$ & $23,3 \%$ & $19,4 \%$ & $30,9 \%$ & $14,6 \%$ \\
\hline & $\begin{array}{l}\text { Empresas de consultoria e consultores } \\
\text { independentes }\end{array}$ & $15,2 \%$ & $6,0 \%$ & $17,8 \%$ & $16,5 \%$ & $4,9 \%$ \\
\hline & Universidades e institutos de pesquisa & $5,9 \%$ & $6,0 \%$ & $6,1 \%$ & $11,2 \%$ & $51,2 \%$ \\
\hline & $\begin{array}{l}\text { Centros de capacitação profissional } \\
\text { e assistência técnica }\end{array}$ & $24,4 \%$ & $6,6 \%$ & $13,9 \%$ & $8,8 \%$ & $12,2 \%$ \\
\hline & $\begin{array}{l}\text { Instituições de testes e ensaios } \\
\text { e certificações }\end{array}$ & $15,0 \%$ & $7,4 \%$ & $15,6 \%$ & $\mathbf{5 , 7 \%}$ & $17,1 \%$ \\
\hline & Licenças, patentes e Know-how & $4,9 \%$ & $3,9 \%$ & $10,1 \%$ & $8,7 \%$ & $4,9 \%$ \\
\hline & $\begin{array}{l}\text { Conferências, encontros e publicações } \\
\text { especializadas }\end{array}$ & $32,8 \%$ & $15,6 \%$ & $15,0 \%$ & $20,1 \%$ & $46,3 \%$ \\
\hline & Feiras e exposições & $39,6 \%$ & $37,3 \%$ & $14,4 \%$ & $19,3 \%$ & $26,8 \%$ \\
\hline & Redes de informação informatizadas & $37,2 \%$ & $36,9 \%$ & $57,8 \%$ & $\mathbf{5 7 , 8 \%}$ & $53,7 \%$ \\
\hline
\end{tabular}

Figura 7 - Empresas Participantes do PINTEC 2005 que Implementaram Inovações, Considerando as Fontes de Informação Empregadas e o Grau de Importância Atribuído pelas Empresas às Fontes

Fonte: Softex (2009, p. 106).

O que nos mostram, em resumo, esses dados e informações sobre a $\mathrm{P} \& \mathrm{D}$ em serviços? Guimarães (2011) registra que a atividade contínua de P\&D, no Brasil, tem sido pouco usual, em especial nas empresas privadas - embora seja maior nas IBSS do que nas demais empresas, conforme mostra a Figura 8. As empresas brasileiras se utilizam pouco de departamentos internos de $\mathrm{P} \& \mathrm{D}$ como fontes internas para a inovação - provavelmente, porque não possuem departamentos formalizados com esta finalidade - e, em relação às fontes externas, também se registra baixo uso de fontes como Universidades e institutos de pesquisa (SOFTEX, 2009).

Esses são indicadores adotados para o setor de serviços de software que, na essência, consistem em indicadores tradicionais de inovação e, portanto, originários de medidas de aplicação industrial - contexto que geralmente tem departamentos formalizados de P\&D. Esta é uma realidade distinta daquela encontrada em serviços, o que exige estabelecer parâmetros mais amplos para a percepção e o diagnóstico de uso de P\&D. Para este estudo, portanto, adotase visão abrangente em relação às possíveis manifestações de $\mathrm{P} \& \mathrm{D}$ em serviços, considerando seu possível caráter não formalizado, não planejado e não restrito a áreas organizacionais tradicionais. Deste modo, entende-se que quaisquer manifestações relativas a construção de conhecimentos nos casos estudados nos interessam como possíveis indicadores de P\&D em serviços.

E que papel se pode esperar, em serviços, para os clientes na condição de inputs de inovação? Certamente, assim como ocorre para $\mathrm{P} \& \mathrm{D}$, também para os clientes há atribuições diferenciadas. Desde a década de 1960, com o início a $2^{a}$ Geração do processo de inovação, tem-se o auge da abordagem demand-pull ou "market-pull" (ROTHWELL, 1994), momento em que se mantem a linearidade dos modelos, mas se introduz ordem distinta da geração precedente (NIOSI, 1999). Enfatiza-se um processo de inovação que busca atender às demandas 
dos consumidores, atribuindo à $\mathrm{P} \& \mathrm{D}$ papel meramente reativo. Assim, os modelos das abordagens demand-pull priorizam o papel do mercado consumidor - e, para serviços, dos clientes - como indutores da inovação. Demarca-se, por fim, que os clientes são "atores de grande importância" no processo de inovação, pois representam fontes de informação. Em alguns casos, "a relação entre fornecedores de serviços e seus clientes pode ser considerada um laboratório em que a inovação é coproduzida (SUNDBO; GALLOUJ, 1998, p. 9). Em serviços, a inovação "surge como uma interação, processo de coprodução e, portanto, com fortes elementos de aprendizagem interativa entre fornecedores e clientes" e é realizada a partir das "relações específicas com o cliente" (HAUKNES, 1998, p. 30).

\section{Método}

Para a responder ao objetivo inicial adotado neste estudo, realizaram-se pesquisas bibliográfica, documental e de campo. A pesquisa bibliográfica se deu por meio da análise de artigos de periódicos científicos indexados a partir do portal de Periódicos da CAPES (SAGE Journal on line; JSTOR Arts \& Science Collection; Springer Link; Emerald and Oxford Journals; Scielo), do Spell.org e do ProQuest. Utilizaram-se como palavras-chave combinações dos seguintes termos para as buscas: inovação; serviços; inovação em serviços; gestão da inovação; processo de inovação e suas respectivas traduções para inglês. A pesquisa documental analisou documentos e publicações originários dos casos selecionados.

Para a pesquisa de campo, desenvolveu-se estudo descritivo, de abordagem qualitativa e com uso de estudo de caso como ferramenta analítica. Opta-se por estudo de casos múltiplos com dez unidades de análise, representadas por dez empresas desenvolvedoras de serviços de software, selecionadas a partir da lista de cadastradas no Sindicato das Indústrias da Informação e Comunicação do Distrito Federal - SINFOR-DF. Como critério de seleção, exigiu-se que as empresas mantivessem em seu portfólio serviços de software típicos, como Consultoria, Treinamento, Suporte, Data-entry, Processamento de Dados, Desenvolvimento e Manutenção de Conteúdos de Páginas da Internet, Integração de Sistemas, Outsourcing, Business Process Outsourcing (BPO) e Software sob Encomenda.

Em cada empresa, foram selecionados especialistas em desenvolvimento de novos serviços ou função correspondente. Para a coleta de evidências, realizaram-se entrevistas presenciais individuais guiadas por um roteiro de entrevista semiestruturado desenvolvido com base nas orientações de Flick (2009) e Richardson (2007). Como estratégia para a análise, utilizou-se análise de conteúdo com codificação temática para construção das categorias de análise definidas a priori (FLICK, 2009). Mobilizaram-se as técnicas de Bardin (2010). As categorias de análise utilizadas são: Identificação da estrutura da empresa para inovação; Participação do cliente na inovação; Participação de Pesquisa e Desenvolvimento (P\&D) na inovação; e Visão acerca do processo de inovação. Na sequência, apresentam-se os resultados por categoria.

\section{Sumarização dos resultados por categorias de análise}

Em relação à Identificação da estrutura para inovação, primeira categoria de análise, os relatos apontam sua baixa formalização nas empresas. Apenas um dos casos estudados apresentou etapas formais definidas especificamente para o processo de gestão da inovação. Embora seja o caso que mais avançou na apresentação de estrutura formal, ainda assim se aponta a não existência de um departamento dedicado à inovação. Nos demais casos, não havia estrutura formal para a inovação. 
Quanto à Participação do cliente na inovação, as empresas foram unânimes em reconhecer a importância do cliente como fonte para a inovação, embora o grau de relevância a ele atribuído pelos entrevistados tenha variações. Apenas uma das dez empresas aponta não ter a prática de envolver o cliente em seu processo de inovação. Quanto à captura das necessidades, ideias e opiniões dos clientes para definir e especificar um novo software, ocorre, principalmente, de duas formas: mantendo funcionários alocados no cliente ou realizando reuniões frequentes com os clientes. Registra-se, portanto, a importância da proximidade geográfica com o cliente durante o desenvolvimento de um software.

No tocante à Participação de P\&D na inovação, os entrevistados foram unânimes em confirmar sua importância como fonte de inovação. Quanto à sua forma de realização, apenas uma empresa apresentou departamento específico para essa atividade, sendo composto por seis pessoas. Em apenas outros três casos os esforços dedicados às atividades de P\&D foram considerados relevantes. A principal justificativa apresentada para a falta de departamentos de P\&D na maior parte das demais empresas é a estrutura enxuta. Quanto aos principais investimentos nas atividades de $\mathrm{P} \& \mathrm{D}$, treinamentos e capacitação de pessoal recebem a maior parte dos recursos. Registra-se, ainda, que os entrevistados apontam que as atividades de P\&D são realizadas de forma contínua e não somente para demandas específicas.

A categoria Visão acerca da inovação revela diversidade nas fontes de inovação relatadas. Destaca-se como fonte de inovação o cliente, que aparece em seis dos casos. P\&D, clientes, funcionários, literatura, mercado, concorrentes, funcionários e Internet são outras fontes de inovação relatadas. Apenas um dos casos faz uso de um centro de pesquisa como fonte para suas inovações. Quanto à existência de sistemas de incentivo e recompensa às atividades inovadoras desenvolvidas por funcionários, apenas três dos casos relatam possuir sistemas de recompensas a funcionários criativos. Quanto ao momento de ocorrência das atividades de $\mathrm{P} \& \mathrm{D}$, relata-se que, em seis dos dez casos, a fase inicial do processo de inovação é a mais intensa no uso de conhecimentos acumulados. Apenas três empresas não possuem participação em redes de cooperação. Entre os parceiros mais comuns, apontam-se outras empresas do ramo, empresas júniores de consultoria, empresas estrangeiras, outras empresas do Arranjo Produtivo Local e incubadoras. Cinco dos casos não fazem uso de políticas públicas para incentivo à inovação. A Figura 8 apresenta a consolidação dos resultados obtidos com as entrevistas realizadas. 


\begin{tabular}{|c|c|c|c|c|c|c|c|c|c|c|}
\hline Empresa & Caso A & Caso B & Caso C & Caso D & Caso $\mathrm{E}$ & Caso $\mathrm{F}$ & Caso $\mathbf{G}$ & Caso $\mathrm{H}$ & Caso I & Caso J \\
\hline Longevidade & 7 anos & 21 anos & 10 anos & 17 anos & 14 anos & 17 anos & 7 anos & 7 anos & 6 anos & 2 anos \\
\hline Funcionários & 90 & 50 & 200 & 21 & 120 & 50 & 40 & 27 & 50 & 14 \\
\hline Principais clientes & Setor Público & Setor Público & Setor Público & Setor Privado & Setor Público & Setor Público & Setor Público & Setor Púlico & Setor Púlico & \begin{tabular}{|l} 
Setor \\
Privado
\end{tabular} \\
\hline $\begin{array}{l}\text { Estrutura formal } \\
\text { para a inovação }\end{array}$ & Não possui & Não possui & Não possui & Não possui & Possui & Não possui & Não possui & Não possui & Não possui & $\begin{array}{l}\text { Não } \\
\text { possui }\end{array}$ \\
\hline $\begin{array}{l}\text { Cliente como fonte } \\
\text { de inovação }\end{array}$ & Sim & Sim & Sim & Sim & Sim & Sim & Sim & Sim & Sim & Sim \\
\hline \begin{tabular}{|l|} 
Importância do \\
cliente na inovação
\end{tabular} & Elevada & Elevada & Elevada & Elevada & Elevada & Elevada & Elevada & Elevada & Elevada & Elevada \\
\hline $\begin{array}{l}\text { Forma de } \\
\text { participação do } \\
\text { cliente }\end{array}$ & \begin{tabular}{|l|} 
Relacionamento \\
próximo com o \\
cliente / Help \\
desk
\end{tabular} & $\begin{array}{l}\text { Atendimento } \\
\text { direto }\end{array}$ & \begin{tabular}{|l|} 
Relacionamento \\
direto com o \\
cliente
\end{tabular} & \begin{tabular}{|l|} 
Atendimento \\
direto e \\
indireto
\end{tabular} & $\begin{array}{l}\text { Relacionamento } \\
\text { constante e } \\
\text { direto }\end{array}$ & $\begin{array}{l}\text { Feedback em } \\
\text { cada etapa da } \\
\text { prestação do } \\
\text { serviço }\end{array}$ & \begin{tabular}{|l|} 
Relaciona- \\
mento direto \\
e constante
\end{tabular} & \begin{tabular}{|l|} 
Relação \\
direta com o \\
cliente
\end{tabular} & \begin{tabular}{|l|} 
Relação \\
direta com o \\
cliente
\end{tabular} & $\begin{array}{l}\text { O cliente } \\
\text { não possui } \\
\text { participa- } \\
\text { ção direta }\end{array}$ \\
\hline $\begin{array}{l}\text { P\&D como fonte } \\
\text { de inovação }\end{array}$ & Sim & Sim & Sim & Sim & Sim & Sim & Sim & Sim & Sim & Sim \\
\hline \begin{tabular}{|l} 
Atividades de $P \& D$ \\
realizadas \\
(principais)
\end{tabular} & Treinamento & $\begin{array}{l}\text { Treinamentos, } \\
\text { equipamentos }\end{array}$ & Treinamentos & Treinamento & Treinamento & Treinamento & Treinamento & Treinamento & Treinamento & $\begin{array}{l}\text { Materiais } \\
\text { e } \\
\text { treinamen } \\
\text { to }\end{array}$ \\
\hline $\begin{array}{l}\text { Momento de } \\
\text { maior uso de P\&D }\end{array}$ & Início & Início & Constante & $\begin{array}{l}\text { Pré-venda e } \\
\text { pós-venda }\end{array}$ & $\begin{array}{l}\text { Momentos } \\
\text { finais da } \\
\text { inovação }\end{array}$ & Início & Contínua & Início & Início & $\begin{array}{l}\text { Validação } \\
\text { da solução } \\
\text { (final) }\end{array}$ \\
\hline $\begin{array}{l}\text { Momento de } \\
\text { maior contribuição } \\
\text { do cliente }\end{array}$ & Início & $\begin{array}{l}\text { Não soube } \\
\text { informar }\end{array}$ & Início & Início & Constante & Constante & Constante & Constante & Constante & \begin{tabular}{|l} 
Validação \\
do \\
problema \\
(início)
\end{tabular} \\
\hline $\begin{array}{l}\text { Principais fontes } \\
\text { de inovação }\end{array}$ & $\begin{array}{l}\text { P\&D, clientes e } \\
\text { funcionários }\end{array}$ & $\begin{array}{l}\text { Cliente e } \\
\text { literatura }\end{array}$ & $\begin{array}{l}\text { Clientes e } \\
\text { mercado }\end{array}$ & \begin{tabular}{|l|} 
Concorrentes \\
e clientes
\end{tabular} & $\begin{array}{l}\text { Funcionários, } \\
\text { clientes }\end{array}$ & $\begin{array}{l}\text { Funcionário e } \\
\text { clientes }\end{array}$ & $\begin{array}{l}\text { Funcionário } \\
\text { e clientes }\end{array}$ & Internet & $\begin{array}{l}\text { Sócios da } \\
\text { empresa }\end{array}$ & $\begin{array}{l}\text { Funcioná- } \\
\text { rios, } \\
\text { clientes e } \\
\text { institutos } \\
\text { de } \\
\text { pesquisa. }\end{array}$ \\
\hline
\end{tabular}

Figura 8 - Resultados Consolidados

Fonte: dados da pesquisa.

\section{Discussão dos resultados}

Os relatos obtidos com as entrevistas trazem revelações valiosas quanto ao papel do cliente e das atividades de P\&D no processo de inovação. Em todos os casos, os entrevistados reconhecem prontamente a participação do cliente como fator crucial para a geração de inovações em serviços de software. Na prática, este seria um indicativo de que os modelos classificados por Rothwell (1994) como de $2^{a}$ geração - oriundos da abordagem market-pull, que posiciona o mercado consumidor como principal indutor de inovações - seriam bons representantes para a percepção dos entrevistados. O mesmo se observa para a P\&D. Quando questionados sobre a importância atribuída às atividades de $\mathrm{P} \& \mathrm{D}$, os especialistas também defenderam, de forma unanime, seu papel imprescindível na geração de inovações, confirmando as premissas de modelos de primeira geração (ROTHWELL, 1994) representantes da abordagem science-push.

Sendo P\&D e participação dos clientes reconhecidos como fundamentais, é de se esperar que estejam presentes no dia-a-dia das organizações, atuando como efetivos inputs de inovação. No entanto, os resultados mostram dissonância entre a importância atribuída pelos entrevistados e o efetivo uso dessas práticas nas organizações. Ou seja: no discurso, clientes e P\&D são rapidamente apontados como cruciais para a geração de inovações. Na prática, os relatos apontam que seus papeis são limitados nas práticas diárias para o desenvolvimento de novos serviços de software. 
Em relação à participação dos clientes, por exemplo, apenas metade das organizações revela ter o hábito de buscar interações constantes com os clientes durante o desenvolvimento de um software. Nos demais casos, essa participação ocorre de forma pontual e em etapas préprogramadas, indicando que as organizações se esforçam para reduzir os espaços de interação com os clientes - reduzindo, assim, o espaço para o surgimento de inovações induzidas por clientes. Esta prática contraria a própria lógica de prestação de serviços, em que se faz uso das interações para desenvolver soluções individualizadas e coproduzidas (GADREY; GALLOU; WEINSTEIN, 1994; HAUKNES, 1998). Ou seja: no discurso, posiciona-se o cliente como peça fundamental no processo de inovação; na prática, restringe-se sua participação para evitar muitas alterações de escopo ou custo no produto final. Essa dualidade fica explícita na fala de um dos entrevistados: "os clientes podem ser facilitadores quando apresentam a demanda de forma clara, nos dão acesso a informações importantes para prestarmos o serviço (...), [mas] alguns clientes dificultam, principalmente em órgãos públicos que designam pessoas sem a devida formação para tratar com os nossos funcionários. Com isso, a comunicação fica mais difícil, além do risco de no final o cliente dizer que não era aquilo que ele queria". Por fim, uma fala, em especial, representa exatamente a dualidade entre o papel real do cliente no processo de inovação e sua importância: "o cliente não tem envolvimento direto no processo de inovação, mas é sim fundamental".

Este relato evidencia, também, outra preocupação frequentemente relatada nos casos: baixa capacidade do cliente para apresentar suas necessidades reais a serem convertidas em requisitos de programação, conforme expressa a seguinte fala - "muitas vezes os usuários dos serviços não conseguem deixar clara a necessidade que possuem”, gerando retrabalhos e aumentos de custos. Como resposta, algumas organizações têm adotado mecanismos para aumentar o nível de correspondência entre a expectativa do cliente e o software final desenvolvido, conforme relata o entrevistado: "a gente só trabalha junto com o cliente [...]. Vir pra cá e ficar aqui é exceção, [...] a grande maioria [dos funcionários] está no cliente, eu tenho provado por ' $a$ ' mais ' $b$ ' que quanto mais gente ficar aqui, mais potencializa o risco de dar algo errado na prestação do serviço [...]".

Ainda em relação à participação dos clientes no processo de inovação, cabe relatar um último obstáculo apresentado nos casos estudados, que se refere à dificuldade em atingir os clientes e os possíveis usuários do software: "nós precisamos do contato com o cliente e com o usuário, que podem ser diferentes. Por exemplo, o cliente e o usuário do Face book são diferentes, o cliente é quem anuncia na página e paga pra isso, o usuário é quem coloca foto, bate papo. Muitas vezes sua missão é juntar os dois, encontrar uma necessidade de um potencial usuário e quem esteja disposto a financiar isso". Essa discrepância é especialmente notória nos casos em que os clientes são organizações públicas, situação em que sua distância em relação aos reais usuários do serviço se torna potencializada.

No tocante à realização de $\mathrm{P} \& \mathrm{D}$, apenas um caso relata ter departamento estruturado para essas atividades - embora todos os casos sejam unânimes em reconhecer o papel da P\&D na indução de inovações. A ausência de departamentos formalizados para essa finalidade não significa, no entanto, que as demais empresas não realizem essas atividades. Na verdade, todas as empresas relatam ter feito investimentos em treinamento de pessoal como evidência de realização de $\mathrm{P} \& \mathrm{D}$. Um caso relata, inclusive, a geração de uma universidade corporativa. Nos casos estudados, é possível reconhecer os elementos característicos do desenvolvimento de P\&D: "projetos com orçamento e objetivos específicos" que "podem ser desenvolvidos por um departamento interno à empresa, específico para tal fim, como também por outros departamentos" (IBGE, 2009). Uma fala se mostra especialmente significativa em relação à natureza dos investimentos em P\&D realizados pelas organizações: "os maiores investimentos em $P \& D$ estão muito ligados ao trabalho intelectual, em treinamento, nos salários, já que a 
mão de obra é altamente especializada, e equipamentos, pois a codificação de software exige maquinário pesado".

Os entrevistados apontam, em sua maioria, que as atividades de P\&D ocorrem de forma contínua nas empresas, embora os relatos apontem sua realização de forma não-sistemática. Segundo a maior parte dos entrevistados, é no momento inicial do processo de inovação que ocorre maior consumo de $\mathrm{P} \& \mathrm{D}$ - aponta-se, portanto, concentração dos esforços de $\mathrm{P} \& \mathrm{D}$ nos casos estudados nas etapas iniciais do desenvolvimento de um software. Essa característica mostra que, na prática, as atividades de $\mathrm{P} \& \mathrm{D}$ ocorrem de forma distinta daquela descrita no modelo linear de inovação discutido por Viotti e Macedo (2003) Brem e Voigt (2009) e Guimarães (2011) - que relatam P\&D como atividades independentes da existência de demanda prévia para os novos produtos ou serviços a serem desenvolvidos. Relata-se, assim, discrepância em relação aos modelos teóricos de primeira geração relatados por Rothwell (1994) - "technology-push". Nos casos estudados, as atividades de P\&D desempenham papel dependente da indução por lacunas de mercado, aproximando-se dos modelos de segunda e terceira geração (ROTHWELL, 1994) e servindo, portanto, como ferramenta para avançar a capacidade de resposta ao mercado, conforme mostra o relato: "certas demandas obrigam a empresa a fazer aprendendo e aprender fazendo, sobretudo quando se trata de uma demanda de que envolve uma tecnologia nova, que a empresa ainda não trabalhe, ou que planejava trabalhar futuramente".

Por fim, cabe apontar o baixo nível de formalização do processo de gestão da inovação relatado pelas empresas. A maior parte das organizações não adota metodologias ou modelos específicos para este fim. A ausência de modelos estruturados implica em perda de memória organizacional e retrabalho, tendo em vista a não-existência de práticas de catalogação de soluções previamente desenvolvidas, conforme se infere a partir da seguinte fala: "[isso nos] impede de replicar as soluções desenvolvidas, [embora] muitas vezes, dois clientes possuam demandas iguais, em momentos diferentes, desenvolvemos a solução para um cliente, estudamos a tecnologia, elaboramos, criamos ferramentas e implementamos. Se, no mês seguinte, outro cliente demandar um software ou um método semelhante, o processo será feito desde o início para atendê-lo".

Tem-se, portanto, a caracterização do processo de gestão da inovação nos casos estudados como de baixa formalização e com altos índices de retrabalho, e sendo muitas vezes disparado por fontes não tradicionais, conforme mostram as falas: "A principal fonte de ideias para inovações vem dos próprios sócios, e a partir daí nós não temos um padrão para transformar a inspiração em produto acabado ou num serviço", ou ainda na seguinte fala: "acontece muitas vezes de uma equipe sair do escritório e ir pra rua pra saber qual é a necessidade das pessoas, ou seja, saber o que eles querem. Isso pode ser um problema a ser resolvido".

\section{Conclusões}

Este estudo investigou o papel desempenhado pelas atividades de $\mathrm{P} \& \mathrm{D}$ e pelos clientes na geração de inovações em empresas desenvolvedoras de serviços software no Distrito Federal. Realizou-se um estudo de casos múltiplos com dez unidades de análise, correspondentes a dez empresas. Coletaram-se evidências com a realização de entrevistas semiestruturadas com especialistas. Para as análises, foram mobilizadas técnicas de análise de conteúdo com codificação temática.

Foram recuperados os principais modelos teóricos adotados para representar o processo de gestão da inovação desde a década de 1940, aqui sumarizados de acordo com o trabalho de 
Rothwell (1994). Os modelos evidenciaram os papeis fundamentais exercidos por clientes e atividades de $\mathrm{P} \& \mathrm{D}$ como indutores de inovações - razão pela qual esses dois elementos foram selecionados para investigação empírica. Desta forma, investigaram-se os papeis teórico atribuídos em cada geração de modelos para clientes e P\&D para, na sequência, confrontá-los com as percepções de gestores e práticas organizacionais.

Os resultados mostraram que os entrevistados reconhecem prontamente a participação do cliente como crucial para a geração de inovações, embora as práticas diárias das organizações revelem a existência de fatores limitadores à efetividade desta participação. $\mathrm{O}$ mesmo ocorre quando os entrevistados são questionados quanto à importância das atividades de P\&D no dia-a-dia das organizações. Aponta-se, portanto, dissonância entre o discurso dos entrevistados e as práticas organizacionais de mobilização dos dois inputs de inovação investigados neste estudo.

No tocante ao papel desempenhado por clientes quanto à indução de inovações em serviços de software, destacam-se os principais resultados apontados nos casos estudados. Apenas metade das organizações buscam interações constantes com os clientes durante o desenvolvimento de um software, sendo que as demais optam por estabelecer participações pontuais e pré-programadas para reduzir os espaços de interação com os clientes como forma de minimizar a incidência de alterações de escopo ou de custo no produto final. Apontou-se, também, um obstáculo à inserção do cliente no processo de desenvolvimento de um software pelas organizações contratadas. Relatos mostram que os clientes possuem baixa capacidade de especificar corretamente suas necessidades em termos de sistemas, levando a retrabalhos e aumentos de custos. Por fim, as organizações relatam dificuldades para atingir os possíveis usuários de um software - que, nem sempre, correspondem ao cliente contratante - levando à redução do potencial de difusão para um software inicialmente contratado.

Em relação ao papel efetivamente desempenhado pelas atividades de $\mathrm{P} \& \mathrm{D}$ na gestão da inovação, sua ocorrência se mostrou presente em todos os casos estudados, de forma mais ou menos estruturada. Todos os casos relatam ter feito investimentos em treinamento de pessoal aqui entendido como o principal indicador de realização de $\mathrm{P} \& \mathrm{D}$, dada a natureza do serviço de software, altamente dependente de capacidade intelectual. Os resultados apontam que as atividades de $\mathrm{P} \& \mathrm{D}$ se apresentaram, em geral, majoritariamente não estruturadas em um departamento específico, não formalizadas e não sistemáticas, tendo em vista a prática de não adotar modelos ou metodologias para gestão do processo de inovação - contrariando a premissa inicial descrita no modelo linear de inovação.

Em suma, os resultados permitiram caracterizar o processo empírico de gestão da inovação efetivamente adotado nos casos como sendo de baixa formalização, baixa estruturação e com altos índices de retrabalho, sendo muitas vezes disparado por fontes não tradicionais. Ressalta-se como principal contribuição do artigo o apontamento da dissonância entre o discurso dos entrevistados sobre a importância de clientes e P\&D e o efetivo papel desempenhado por esses inputs nas práticas organizacionais. Enquanto a importância atribuída pelos entrevistados corresponde aos papeis inicialmente admitidos para clientes e P\&D nos modelos de primeira e segunda geração sumarizados por Rothwell (1994), as práticas realmente exercidas para a inserção de clientes e de $\mathrm{P} \& \mathrm{D}$ como insumos para a inovação se afasta dos modelos. Forma-se, assim, uma observação curiosa: os modelos teóricos embasam o discurso dos entrevistados; mas não as práticas organizacionais.

Como limitações para a presente pesquisa, destacam-se a limitação geográfica - já que apenas empresas do DF foram consideradas - e o recorte de atividade, que limitou nossa análise exclusivamente ao segmento dos serviços de software. Como recomendações para estudos futuros, recomendam-se ampliações de escopo geográfico e de atividades no setor de serviços. 


\section{REFERÊNCIAS}

BARDIN, L. Análise de conteúdo. Lisboa: Edições 70, 2010.

BARRAS, R. Towards a theory of innovation in services. Research policy, v. 15, n. 4, p. 161173, 1986.

BESSANT, J.; TIDD, J. Inovação e Empreendedorismo. Porto Alegre: Bookman, 2009.

BREM, A.; VOIGT, K. I. Integration of market pull and technology push in the corporate front end and innovation management - Insights from the German software industry. Technology Management in the Service Economy, v. 29, n. 5, p. 351-367, mai. 2009.

CAMPOS, R. R.; NICOLAU, J. A.; SIMIONI, M. Inovação e interação produtor-usuário nas empresas de software. In: SBRAGIA, R.; STAL, E. (Orgs.) Tecnologia e Inovação. São Paulo: PGT/USP, 2002.

DORNER, N.; GASSMANN, O.; GEBAUER, H. Service innovation: why is so difficult to accomplish? Journal of Business Strategy, v. 32, n. 3, p. 37-46. 2011.

FLICK, U. Introdução à pesquisa qualitativa. $3^{\text {a }}$ Porto Alegre: Artmed, 2009.

FORAY, D.; LUNDVALL, B. The knowledge-based economy: from the economics of knowledge to the learning economy. The economic impact of knowledge, p. 115-121, 1998.

GADREY, J. Emprego, produtividade e avaliação do desempenho dos serviços. In SALERNO, M. (Org.). Relação de serviço: produção e avaliação. (pp. 23-66). São Paulo: Editora Senac, 2001.

GALLOUJ, F. Innovation in the service economy: the new wealth of nations. Cheltenham, UK: Edward Elgar, 2002.

GALLOUJ, F.; SAVONA, M. Innovation in services: a review of the debate and a research agenda. Journal of Evolutionary Economics, v. 19, n. 2, p. 149-172. 2008.

GALLOUJ, F.; WEINSTEIN, O. Innovation in services. Research policy, v. 26, n. 4, p. 537 $556,1997$.

GODIN, B. The Linear model of innovation the historical construction of an analytical framework. Science, Technology \& Human Values, v. 31, n. 6, p. 639-667, 2006.

GUIMARÃES, S. K. Empreendedorismo intensivo em conhecimento no Brasil. Cad. CRH [online], Salvador, v. 24, n. 63, p. 575-592, dez. 2011.

HANSEN, M. T.; BIRKINSHAW, J. The innovation value chain. Harvard Business Review, jun. 2007.

HAUKNES, J. Services in innovation - Innovation in services. SI4S Final report, p. 1-101, 1998. 
IBGE - Instituto Brasileiro de Geografia e Estatística. Pesquisa de Inovação Tecnológica PINTEC (2008): Instruções para o preenchimento do questionário. Rio de Janeiro: IBGE, 2009.

KLINE, S. J. Rosenberg. N., 1986. An overview of innovation. The positive sum strategy: Harnessing technology for economic growth, p. 275-306, 1986.

KUBOTA, L. C. As Kibs e a inovação tecnológica das firmas de serviços. Economia e Sociedade, Campinas, v. 18, n. 2, p. 349-369, ago. 2009.

NELSON, R.; WINTER, S. Uma teoria evolucionária da mudança econômica. Editora Unicamp, 2005.

NIOSI, J. Fourth-Generation R\&D: From Linear Models to Flexible Innovation. Journal of Business Research, New York, v. 45, n. 2, p. 111-117, jun. 1999.

OECD. Proposed Standard Practice for Surveys on Research and Experimental Development, Frascati Manual. França: OECD Publications Service, 2002.

OKE, A. Innovation types and innovation management practices in service companies. International Journal of Operations \& Production Management, USA, v. 27, n. 6, p. 564587. 2007.

PORTER, M. Competitive advantage: Creating and sustaining superior performance. Simon and Schuster, 2008.

RENESER. Research and Development Needs of Business Related Services Firms. Dialogic Group. Utrecht. Set. 2006.

RICHARDSON, R. Pesquisa Social: Métodos e Técnicas. Editora Atlas. 3.ed. São Paulo, 2007.

ROTHWELL, R. Towards the fifth-generation innovation process. International Marketing Review, UK, v. 11, n. 1, p. 7-31. 1994.

RUBALCABA, L. The new service economy. Challenges and Policy Implications for Europe. Elgar, Cheltenham, UK, 2007.

SALERNO, M. (Org.). Relação de serviço: produção e avaliação. (pp. 23-66). São Paulo: Editora Senac, 2001.

SAWATANI, Y.; FUJIGAKI, Y. Service R\&D Program Design Aiming at Service Innovation. In: Service Systems Science. Springer Japan, 2015. p. 163-174.

SCHUMPETER, J. A. Teoria do desenvolvimento econômico: uma investigação sobre lucros, capital, crédito, juro e o ciclo econômico. São Paulo: Nova Cultura, 1997. Original: 1942. 
SOFTEX - SOCIEDADE BRASILEIRA PARA PROMOÇÃO DA EXPORTAÇÃO DE SOFTWARE. Software e Serviços de TI: A indústria brasileira em perspectiva - 2009. Brasil: Observatório SOFTEX. Campinas: 2009.

SUNDBO, J.; GALLOUJ, F. Innovation in Services. SI4S Project synthesis. Step Group, 1998. Step Group, Work package 3/4.

TIDD, J.; BESSANT, J.; PAVITT, K. Gestão da inovação. Porto Alegre: Bookman, 2008.

VARGAS, E.; BOHRER, C.; FERREIR, L.; MOREIRA, M. A Pesquisa Sobre Inovação em Serviços no Brasil: Estágio Atual, Desafios e Perspectivas. REGEPE-Revista de Empreendedorismo e Gestão de Pequenas Empresas, v. 2, n. 1, 2013.

VASCONCELLOS, L. H. R.; MARX, R. Como ocorrem as inovações em serviços? Um estudo exploratório de empresas no Brasil. Gestão \& Produção, São Carlos, v. 18, n. 3, p. 443-460, 2011.

VIOTTI, E; MACEDO, M. (Orgs.). Indicadores de ciência, tecnologia e inovação no Brasil. 01. ed. Campinas: Editora da Unicamp, 2003. 